\title{
Genetic Variability in Bangladeshi Aromatic Rice through RAPD Analysis
}

\author{
Mehfuz Hasan $^{1 *}$, Mohammad Sharif Raihan ${ }^{1}$ \\ 1*Department of Genetics and Plant Breeding, Bangabandhu Sheikh Mujibur Rahman Agricultural University, Salna, Gazipur 1706, \\ Bangladesh.
}

\section{A R T I C LE IN F O}

Article history:

Received 07 July 2014

Accepted 12 August 2014

Available online, ISSN: 2148-127X

Keywords:

Aromatic rice (Oryza sativa L.)

Genetic variation

Polymorphism

Random amplified polymorphic DNA

Un-weighted pair-group

${ }^{*}$ Corresponding Author:

E-mail: saikathu@yahoo.com

\begin{abstract}
A B S T R A C T
Genetic polymorphism and relationships among 30 commercial varieties of Bangladeshi aromatic rice (Oryza sativa L.) were established using random amplified polymorphic DNA (RAPD) primers. Out of fifty 10-mer RAPD primers screened initially, four were chosen and used in a comparative analysis of different varieties of indigenous Bangladeshi aromatic rice. Of the 33 total RAPD fragments amplified, 7 (21.21\%) were found to be shared by individuals of all eight varieties. The remaining 26 fragments were found to be polymorphic (78.79\%). Pair-wise estimates of similarity ranged from 0.101 to 0.911. Highest genetic diversity was determined between Radhunipagol and Dubsail varieties (0.911). The amount of genetic diversity within aromatic rice germplasm was quite high as determined by the genetic similarity coefficients between varieties. Genetic similarities obtained from RAPD data were also used to create a cluster diagram. Cluster analysis using an un-weighted pair-group method with arithmetic averages (UPGMA) was used to group the varieties and the 30 aromatic rice varieties were grouped into 6 clusters where cluster I includes the maximum number of varieties (9). Cluster VI includes minimum number of varieties (2). This Study offered a rapid and reliable method for the estimation of variability between different varieties which could be utilized by the breeders for further improvement of the local aromatic rice varieties.
\end{abstract}

\section{Introduction}

The indigenous aromatic rice germplasm of Bangladesh is enriched with wide genetic diversity and valuable gene system for yield attributes and adaptability. A detailed understanding about the extent structure of genetic variation in different varieties of the same species is important for the development of appropriate and efficient strategies for collection, conservation, and preservation of cultivar relatives. In addition, the efficient use of genetic resources in all plant-breeding programs requires knowledge about genetic diversity. At present, several varieties have many synonyms in different regions which make identification difficult. Nevertheless, differentiation of varieties through morphological features is inefficient and inaccurate (Rahman et al., 2007).

The conservation and characterization of aromatic rice germplasm in Bangladesh is very important. Proper characterization and classification of rice germplasm is imperative for their effective management and subsequent utilizations. Traditionally rice germplasm is characterized based on morphology. Selection of plant varieties based on morphological characters is not very reliable because major characters of interest possess low heritability and are genetically complex. Molecular markers based on DNA sequence are found to be more reliable (Raghunathachari et al., 2000; Karunagoda and Bandara,
2005; Mani et at., 2010; Rasheed, 2005). They represent an opportunity to provide information on the variation that exists in a particular species within a local region as well as among different countries. They serve as a valuable guide for effective collection and use of genetic resources too. Molecular markers provide information that helps in deciding the distinctiveness of species and their ranking according to the number of close relatives and phylogenetic position. Moreover, varietal distinctiveness and relativeness can unambiguously be estimated by DNA fingerprinting in commercially important crops (Thomas et al., 2006).

Recent advances in molecular biology enabled DNAbased markers to study genetic basis of crops (Agarwal, 2003; Liu et al., 2007; Prabakaran et al., 2010; Yasmin et al., 2012). Molecular markers have been developed for rice using randomly amplified polymorphic DNA markers (RAPDs) and microsatellites (Singh, 2003; Abbas, 2000; Das, 2009; Saker et al., 2005; Tehrim et al., 2012; Alessandra et al., 2009; Bhhartiya et al., 2009). Among these DNA fingerprinting methods, use of RAPDs has the advantage that it can be used without prior knowledge of the genome.

There is very little information available on the nature and extent of genetic diversity of Bangladeshi aromatic 
rice, particularly using molecular markers. This information is quite valuable for rationalization of aromatic rice germplasm conservation and their utilization in a breeding program. Therefore, the study aimed to measure variability among Bangladeshi aromatic rice varieties grown under different names throughout the country based on RAPD markers.

\section{Materials and methods}

\section{Plant material}

Thirty indigenous rice varieties collected from different regions of Bangladesh were studied. The seeds of collected varieties were grown in pots. Leaf samples were collected from two-week old plants and for use in DNA assays.

\section{DNA extraction}

Genomic DNA was extracted according to modified CTAB (cetyltrimethylammonium bromide) method described by Doyle and Doyle (1987). Approximately $200 \mathrm{~g}$ of rice leaf tissue was chilled and ground to a very fine powder by mortar and pestle. The powdered tissue was transferred to a $1.5 \mathrm{ml}$ microcentrifuge tube containing $700 \mu \mathrm{l}$ of prewarmed $\left(65^{\circ} \mathrm{C}\right)$ DNA extraction buffer. The contents were incubated for 40 minutes at $65^{\circ} \mathrm{C}$ in shaking water bath. $700 \mu \mathrm{l}$ of chloroform: isoamylalcohol $(24: 1)$ was added for extraction. The mixture was centrifuged for $15 \mathrm{~min}$ at $14000 \mathrm{rpm}$. The aqueous phase was transferred to another fresh tube and isopropanol (2/3 of the aqueous phase) was added and mixed gently to precipitate DNA. The mixture was centrifuged for $20 \mathrm{~min}$ at $14000 \mathrm{rpm}$. The supernatant was discarded and the pellet was washed with $70 \%$ ethanol. The pellet was dried completely and dissolved in TE buffer. The purity and concentration of DNA were determined by using the spectrophotometer at wavelengths of $260 \mathrm{~nm}$ and $280 \mathrm{~nm}$. The working concentration was prepared by diluting DNA to obtain 25 $\mathrm{ng} / \mu \mathrm{l}$ strength.

\section{PCR amplification}

RAPD amplification was performed in a reaction volume of $25 \mu \mathrm{l}$ containing $10 \mathrm{X}$ reaction buffer (100 mMTris, $500 \mathrm{mM} \mathrm{KCl,} 0.1 \%$ gelatin, $\mathrm{pH} 9.0)$ with 1.5 $\mathrm{mM} \mathrm{MgCl} 2,5 \mathrm{pM}$ of primer, $0.2 \mathrm{mM}$ dNTPs (dATP, dCTP, dGTP and dTTP), 2 U Taq DNA polymerase and $25 \mathrm{ng}$ of template DNA.

PCR amplification used the following profile: 45 cycles of $94^{\circ} \mathrm{C}$ for $1 \mathrm{~min}, 35^{\circ} \mathrm{C}$ for $1 \mathrm{~min}$, and $72^{\circ} \mathrm{C}$ for 1 min with an extension of $72^{\circ} \mathrm{C}$ for $10 \mathrm{~min}$. The resulting products were electrophoretically analyzed through $1.5 \%$ agarose gel stained with ethidium bromide $(5 \mu \mathrm{g} / \mathrm{ml})$ in TBE buffer (90 mM Tris-borate and $2 \mathrm{mM}$ EDTA, pH 8.0). After staining in $0.5 \mu \mathrm{g} / \mathrm{ml}$ ethidium bromide solution, gels were photographed on a UV transilluminator. The genomes were scored for presence or absence of polymorphic bands and intensity of these polymorphic bands. Index of genetic variation was calculated and dendrograms were constructed through UPGMA method by using computer software.

Fifty decamer random primers were initially screened for the presence of bands. Out of them, four primers (OPA 14, OPC 07, OPC 15 and OPF 17) were selected as they produced polymorphic and reproducible banding profiles.

\section{Results and discussion}

Fifty decamer operon primers were used in this study. Four primers (Table 1) produced amplicons and they were selected by primary screening and were selected for genetic diversity analysis by taking into consideration the repeatability, sharpness and intensity of bands.

Out of 33 bands generated, 7 (21.21\%) were found to be shared by individuals of all thirty varieties. The remaining 26 fragments were found to be polymorphic (78.79\%). Rahman et al., (2009) reported four primers which gave reproducible and distinct polymorphic amplified products. Selected four primers generated 41 bands where 37 bands $(90.24 \%)$ were polymorphic and 4 bands $(9.76 \%)$ were monomorphic.

In the present study the percentage of polymorphic loci was 78.79. A diverse level of polymorphism in rice genotypes were reported by Qian et al., (2006), 83.5\%, Shivapriya and Shailaja (2006), 74.1\%.

A similarity matrix based on the proportion of shared RAPD fragments was used to establish the level of relatedness between aromatic varieties. Pair-wise estimates of similarity ranged from 0.101 to 0.911 (Table 2 ). Highest genetic diversity revealed in RAPD analysis was determined between Radhunipagol and Dubsail (0.911). The amount of genetic diversity within rice germplasm is quite high as revealed by the genetic similarity coefficients between lines. Kanawapee et al., (2011) also examined 30 rice genotypes and found high genetic diversity among the genotypes. In their experiment pair-wise estimates of genetic similarity among the 30 genotypes ranged from 0.64 to 0.94 .

Genetic similarities obtained from RAPD data were used to create a cluster diagram. Cluster analysis based on Nei's similarity coefficients using UPGMA grouped 30 cultivars into 6 clusters (Fig. 2). Cluster I includes 9 varieties namely Agali, Jirabhog, Bashful, Doiergura, Guamuri, Jesobalam, Kaminisaru, Lalsoru and Premful. This cluster is further differentiated into 2 subclusters with Subcluster I including 3 rice varieties namely Agali, Jirabhog, Bashful and Subcluster II with varieties Doiergura, Guamuri, Jesobalam, Kaminisaru, Lalsoru and Premful. The Cluster II includes 6 varieties namely Uknimadhu, Tilkapur, Sorukamini, Padmabhog, Dubsail and BR5 which was futher differtiated with 2 subclusters. Subcluster I includes Uknimadhu and Tilkapur while Subcluster II includes 4 other varieties namely Sorukamini, Padmabhog, Dubsail and BR5. Cluster III has 5 varieties namely Duksail, Thakurbhog, Chiniatab, Khorma and Rajbhog while Cluster IV consists of 4 varieties namely Kalguchi, Badshahbhog, Dudhkalam and Lalbinni. Cluster V has 5 varieties namely Chinigura Maloti, Chinisakkhor and Kataribhog while VI contains two varieties of Kalijira and Radhunipagol. 
Table 1 Selected primers and genetic variations among rice varieties in RAPD analysis

\begin{tabular}{|c|c|c|c|c|c|c|c|}
\hline \multirow[t]{2}{*}{$\begin{array}{l}\text { Primer } \\
\text { code }\end{array}$} & \multirow[t]{2}{*}{ Sequence } & \multirow[t]{2}{*}{$\begin{array}{c}\text { Total no. of } \\
\text { bands }\end{array}$} & \multirow[t]{2}{*}{$\begin{array}{l}\text { App. Range of } \\
\text { size (bp) }\end{array}$} & \multicolumn{2}{|c|}{$\begin{array}{c}\text { Polymorphic } \\
\text { bands }\end{array}$} & \multicolumn{2}{|c|}{$\begin{array}{l}\text { Monomorphic } \\
\text { bands }\end{array}$} \\
\hline & & & & No. & $\%$ & No. & $\%$ \\
\hline OPA 14 & $5^{\prime}{ }^{\text {TCTGTGCTGG3 }}{ }^{\prime}$ & 9 & $250-1600$ & 6 & 66.67 & 3 & 33.33 \\
\hline OPC-07 & 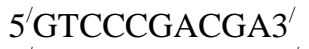 & 8 & $200-1800$ & 7 & 87.50 & 1 & 14.20 \\
\hline OPC-15 & $5^{\prime}$ GACGGATCAG3' & 9 & $400-1300$ & 8 & 88.89 & 1 & 11.11 \\
\hline OPF-17 & $5^{\prime} \mathrm{AACCCGGGAA} 3^{\prime}$ & 7 & $200-1700$ & 5 & 71.43 & 2 & 28.57 \\
\hline Total & & 33 & & 26 & 78.79 & 7 & 21.21 \\
\hline
\end{tabular}
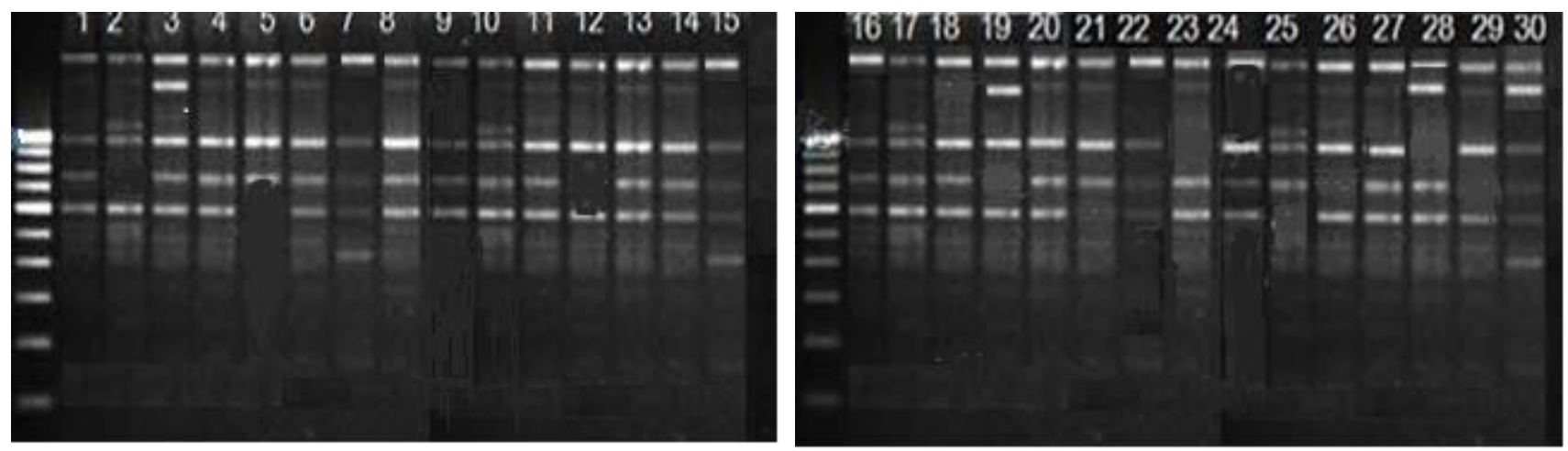

Fig. 1 RAPD profile of 30 aromatic rice varieties generated by OPC 15 primer.

1:Jesobalam, 2:Doiergura, 3:Guamuri, 4:Rajbhog, 5:Agali, 6:Premful, 7:Dubsail, 8:Duksail, 9:Radhunipagol, 10:Kataribhog, 11:Kalguchi, 12:Chinigura, 13:Badshahbhog, 14:Chini atab, 15:Tilkapur, 16:Kaminisaru, 17:Sorukamini, 18:Padmabhog, 19:Thakurbhog, 20:Maloti, 21:Lalbinni, 22:Jirabhog, 23:Dudhkalam, 24:Lalsoru, 25:Chinishakkhor, 26:Bashful, 27:Kalijira, 28:BR5, 29:Uknimadhu, 30:Khorma

Precise identification of crop cultivars is required for variety registration, preventing misappropriation and for protection of plant breeders' as well as farmers' rights. While the debate is going on with regard to acceptability of DNA data for defining distinctiveness, uniformity and stability (DUS) of crop varieties and deciding on the minimum distance for declaring two varieties as different, creation of data base on the morphological characteristics and DNA fragment patterns is widely accepted by all concerned. In the present study, a basic data set was created for aromatic rice using RAPD fingerprints. The total number of amplified fragments and number of polymorphic fragments contained in this data set enabled identification and differentiation of the studied aromatic rice genotypes.

Genetic variation is important in maintaining the developmental stability and biological potential of an organism. In essence, the present work revealed ample genetic variation among the studied aromatic rice germplasm. Significant levels of genetic variation were observed between each pair of rice cultivars which indicate rich genetic resources in aromatic rice of Bangladesh. Information on this genetic variation from the present study might be useful for breeders in making decision for improvement of rice cultivars through selective breeding and cross breeding programs. Besides this, breeders could make a strategy for conservation of cultivars having diverse gene pools. As literature on genetic analysis of Bangladeshi aromatic rice using molecular markers is very scarce, present study could help the researchers in this regard in future.

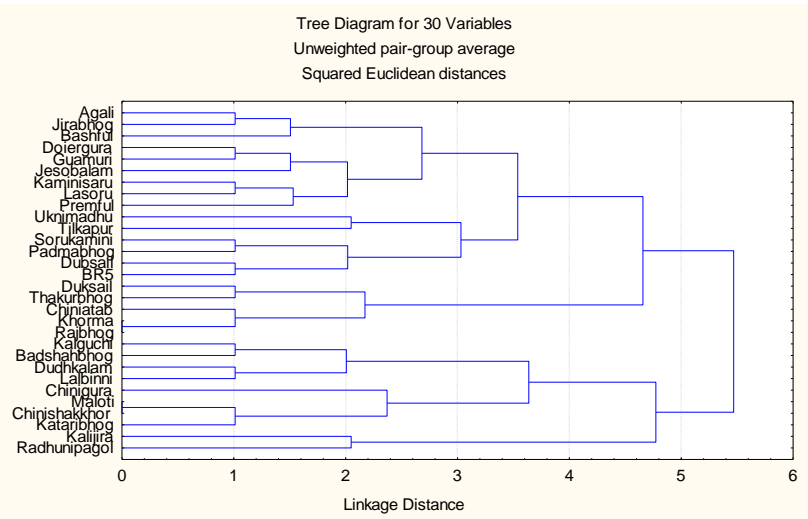

Fig 2 Dendrogram (UPGMA) pattern of RAPD analysis in different aromatic rice varieties.

\section{Acknowledgments}

This work was funded by the Research Management Committee (RMC), Bangabandhu Sheikh Mujibur Rahman Agricultural University, Bangladesh 
Table 2. Similarity matrix of rice varieties analyzed using Nei's original measures of genetic identity

\begin{tabular}{|c|c|c|c|c|c|c|c|c|c|c|c|c|c|c|c|c|c|c|c|c|c|c|c|c|c|c|c|c|c|c|}
\hline VN & 1 & 2 & 3 & 4 & 5 & 6 & 7 & 8 & 9 & 10 & 11 & 12 & 13 & 14 & 15 & 16 & 17 & 18 & 19 & 20 & 21 & 22 & 23 & 24 & 25 & 26 & 27 & 28 & 29 & 30 \\
\hline 1 & 1 & & & & & & & & & & & & & & & & & & & & & & & & & & & & & \\
\hline 2 & 0.2 & 1 & & & & & & & & & & & & & & & & & & & & & & & & & & & & \\
\hline 3 & 0.205 & 0.205 & 1 & & & & & & & & & & & & & & & & & & & & & & & & & & & \\
\hline 4 & 0.101 & 0.301 & 0.301 & 1 & & & & & & & & & & & & & & & & & & & & & & & & & & \\
\hline 5 & 0.101 & 0.301 & 0.311 & 0.205 & 1 & & & & & & & & & & & & & & & & & & & & & & & & & \\
\hline 6 & 0.101 & 0.301 & 0.311 & 0.205 & 0.2 & 1 & & & & & & & & & & & & & & & & & & & & & & & & \\
\hline 7 & 0.101 & 0.301 & \begin{tabular}{|l|}
0.301 \\
\end{tabular} & 0.2 & 0.205 & 0.205 & 1 & & & & & & & & & & & & & & & & & & & & & & & \\
\hline 8 & 0.101 & 0.301 & \begin{tabular}{|l|}
0.311 \\
\end{tabular} & 0.205 & 0.2 & 0.2 & 0.205 & 1 & & & & & & & & & & & & & & & & & & & & & & \\
\hline 9 & 0.2 & 0.2 & 0.205 & 0.301 & 0.301 & 0.301 & 0.301 & 0.301 & 1 & & & & & & & & & & & & & & & & & & & & & \\
\hline 10 & 0.2 & 0.4 & \begin{tabular}{|l|}
0.405 \\
\end{tabular} & $\mid 0.101$ & 0.301 & 0.301 & 0.301 & \begin{tabular}{|l|}
0.301 \\
\end{tabular} & 0.4 & 1 & & & & & & & & & & & & & & & & & & & & \\
\hline 11 & 0.2 & 0.4 & 0.205 & 0.301 & 0.101 & 0.301 & 0.301 & 0.301 & 0.4 & 0.4 & 1 & & & & & & & & & & & & & & & & & & & \\
\hline 12 & 0.2 & 0.4 & 0.405 & 0.301 & 0.301 & 0.101 & 0.101 & 0.301 & 0.4 & 0.4 & 0.4 & 1 & & & & & & & & & & & & & & & & & & \\
\hline 13 & 0.2 & 0.4 & 0.405 & 0.301 & 0.301 & 0.301 & 0.101 & 0.101 & 0.4 & 0.4 & 0.4 & 0.2 & 1 & & & & & & & & & & & & & & & & & \\
\hline 14 & 0.301 & 0.301 & 0.311 & 0.405 & 0.4 & 0.4 & 0.405 & 0.4 & 0.101 & 0.301 & 0.501 & 0.501 & 0.501 & 1 & & & & & & & & & & & & & & & & \\
\hline 15 & 0.301 & 0.301 & \begin{tabular}{|l|}
0.301 \\
\end{tabular} & 0.2 & 0.205 & 0.405 & 0.4 & 0.405 & 0.501 & 0.301 & 0.101 & 0.501 & 0.501 & 0.605 & 1 & & & & & & & & & & & & & & & \\
\hline 16 & 0.301 & 0.501 & 0.511 & 0.405 & 0.4 & 0.2 & 0.205 & 0.2 & 0.501 & 0.501 & 0.501 & 0.101 & 0.101 & 0.6 & 0.605 & 1 & & & & & & & & & & & & & & \\
\hline 17 & 0.4 & 0.4 & 0.405 & 0.301 & 0.501 & 0.501 & 0.501 & \begin{tabular}{|l|}
0.501 \\
\end{tabular} & 0.2 & 0.2 & 0.6 & 0.6 & 0.6 & 0.101 & 0.501 & \begin{tabular}{|l|}
0.701 \\
\end{tabular} & 1 & & & & & & & & & & & & & \\
\hline 18 & 0.4 & 0.4 & 0.405 & 0.501 & 0.301 & 0.301 & 0.301 & \begin{tabular}{|l|}
0.501 \\
\end{tabular} & 0.6 & 0.6 & 0.2 & 0.2 & 0.4 & 0.701 & 0.301 & \begin{tabular}{|l|}
0.301 \\
\end{tabular} & 0.8 & 1 & & & & & & & & & & & & \\
\hline \begin{tabular}{|l|}
19 \\
\end{tabular} & 0.501 & 0.501 & \begin{tabular}{|l|}
0.511 \\
\end{tabular} & 0.405 & 0.4 & 0.6 & 0.605 & 0.6 & 0.301 & 0.301 & 0.501 & 0.701 & 0.701 & 0.2 & 0.405 & 0.8 & 1.01 & 0.701 & 1 & & & & & & & & & & & \\
\hline 20 & 0.501 & 0.501 & \begin{tabular}{|l|}
0.511 \\
\end{tabular} & 0.605 & 0.4 & 0.4 & 0.405 & 0.4 & 0.701 & 0.701 & 0.301 & 0.301 & 0.301 & 0.8 & 0.405 & 0.2 & 9.01 & 0.101 & 0.8 & 1 & & & & & & & & & & \\
\hline 21 & 0.501 & 0.301 & \begin{tabular}{|l|}
0.301 \\
\end{tabular} & 0.4 & 0.405 & 0.605 & 0.6 & 0.905 & 0.301 & 0.501 & 0.301 & \begin{tabular}{|l|l|}
0.701 \\
\end{tabular} & 0.701 & 0.405 & 0.2 & 0.805 & 3.01 & 0.501 & 0.205 & 0.605 & 1 & & & & & & & & & \\
\hline 22 & 0.6 & 0.6 & 0.605 & 0.501 & 0.501 & 0.501 & 0.501 & 0.501 & 0.8 & 0.6 & 0.4 & 0.4 & 0.4 & 0.901 & 0.301 & 0.301 & 0.8 & 0.2 & 0.701 & 0.101 & 0.501 & 1 & & & & & & & & \\
\hline 23 & \begin{tabular}{|l|}
0.701 \\
\end{tabular} & 0.501 & \begin{tabular}{|l|}
0.511 \\
\end{tabular} & 0.605 & 0.6 & 0.6 & 0.805 & 0.8 & 0.501 & \begin{tabular}{|l|}
0.501 \\
\end{tabular} & 0.501 & 0.701 & 0.901 & 0.4 & 0.405 & 0.8 & 3.01 & 0.501 & 0.2 & 0.6 & 0.205 & 0.501 & 1 & & & & & & & \\
\hline 24 & 0.701 & 0.701 & \begin{tabular}{|l|}
0.711 \\
\end{tabular} & 0.605 & 0.6 & 0.6 & 0.605 & 0.6 & 0.901 & 0.501 & 0.501 & 0.501 & 0.501 & 0.8 & 0.405 & 0.4 & 7.01 & 0.301 & 0.6 & 0.2 & 0.605 & \begin{tabular}{|l|}
0.101 \\
\end{tabular} & 0.4 & 1 & & & & & & \\
\hline 25 & 0.701 & 0.501 & \begin{tabular}{|l|}
0.511 \\
\end{tabular} & 0.605 & 0.6 & 0.6 & 0.805 & 0.8 & 0.501 & 0.501 & 0.501 & 0.701 & 0.901 & 0.4 & 0.405 & 0.8 & 3.01 & 0.501 & 0.6 & 0.6 & 0.205 & 0.501 & 0 & 0.4 & 1 & & & & & \\
\hline 26 & 0.701 & 0.701 & \begin{tabular}{|l|}
0.711 \\
\end{tabular} & 0.605 & 0.6 & 0.6 & 0.605 & 0.6 & 0.901 & 0.501 & 0.501 & 0.501 & 0.501 & 0.8 & 0.405 & 0.4 & 7.01 & 0.301 & 0.301 & 0.2 & 0.605 & 0.101 & 0.4 & 0 & 0.4 & 1 & & & & \\
\hline 27 & 0.8 & 0.6 & 0.605 & 0.701 & 0.701 & 0.701 & 0.701 & 0.901 & 0.6 & 0.6 & 0.6 & 0.6 & 0.8 & 0.501 & 0.501 & 0.701 & 0.4 & 0.4 & 0.301 & 0.501 & 0.301 & 0.4 & 0.101 & 0.301 & 0.101 & 0.301 & 1 & & & \\
\hline 28 & 0.4 & 0.4 & 0.405 & 0.301 & 0.301 & 0.301 & 0.501 & 0.501 & 0.6 & 0.4 & 0.2 & 0.4 & 0.6 & 0.701 & 0.101 & 0.501 & 0.6 & 0.2 & 0.501 & 0.301 & 0.301 & 0.2 & 0.301 & 0.301 & 0.301 & 0.301 & 0.4 & 1 & & \\
\hline 29 & 0.4 & 0.4 & 0.405 & 0.501 & 0.501 & 0.501 & 0.301 & 0.301 & 0.2 & 0.6 & 0.6 & 0.4 & 0.2 & 0.301 & 0.701 & 0.301 & 0.4 & 0.6 & 0.501 & 0.501 & 0.501 & 0.6 & 0.701 & 0.701 & $\mid 0.701$ & 0.701 & 0.6 & 0.8 & 1 & \\
\hline 30 & 0.605 & 0.601 & 0.62 & 0.711 & 0.701 & 0.501 & 0.511 & 0.501 & 0.405 & 0.605 & 0.805 & 0.405 & 0.405 & 0.301 & 0.911 & 0.301 & 0.405 & 0.605 & 0.501 & 0.501 & 0.711 & 0.605 & 0.501 & 0.501 & 0.501 & \begin{tabular}{|l|}
0.501 \\
\end{tabular} & 0.405 & 0.805 & 0.205 & 1 \\
\hline
\end{tabular}

Dudhkalam, 18: Duksail, 19: Lalbinni, 20: Thakurbhog, 21: Chiinigura, 22: Chiniatab, 23: Maloti, 24: Khorma, 25: Chinishakkhor, 26: Rajbhog, 27: Kataribhog, 28: BR5, 29: Kalijira, 30: Radhunipagol 
Table 3 Distribution of 30 aromatic rice genotypes in different clusters

\begin{tabular}{|c|c|c|c|}
\hline Cluster & \multicolumn{2}{|c|}{ Genotypes included in different clusters } & No. of genotypes in cluster \\
\hline \multirow{3}{*}{$\mathrm{I}$} & $\begin{array}{l}\text { Agali, Jirabhog, Bashfi } \\
\text { Lalsoru, Premful }\end{array}$ & amuri, Jesobalam, Kaminisaru, & \multirow{3}{*}{9} \\
\hline & \multicolumn{2}{|l|}{ Subcluster } & \\
\hline & Agali, Jirabhog, Bashful & $\begin{array}{l}\text { Doiergura, Guamuri, Jesobalam, } \\
\text { Kaminisaru, Lalsoru, Premful }\end{array}$ & \\
\hline \multirow{2}{*}{ II } & \multicolumn{2}{|c|}{$\begin{array}{l}\text { Uknimadhu, Tilkapur, Sorukamini, Padmabhog, Dubsail, BR5 } \\
\text { Subcluster }\end{array}$} & \multirow{2}{*}{6} \\
\hline & Uknimadhu, Tilkapur & $\begin{array}{l}\text { Sorukamini, } \\
\text { Dubsail, BR5 }\end{array}$ & \\
\hline III & \multirow{4}{*}{\multicolumn{2}{|c|}{$\begin{array}{l}\text { Duksail, Thakurbhog, Chiniatab, Khorma, Rajbhog } \\
\text { Kalguchi, Badshahbhog, Dudhkalam, Lalbinni } \\
\text { Chinigura, Maloti, Chinisakkhor, Kataribhog } \\
\text { Kalijira, Radhunipagol }\end{array}$}} & 5 \\
\hline IV & & & 4 \\
\hline $\mathrm{V}$ & & & 4 \\
\hline VI & & & 2 \\
\hline
\end{tabular}

\section{References}

Abbas ST. 2000. Development of Rachna basmati- a new fine, aromatic rice variety- through biotechnological tools. Sc.Vis., 5: 74-84

Agarwal M, Sahi C, Katiyar-Agarwal S, Agarwal S, Young T, Gallie, DR, Sharma VM, Ganesan K, Grover A. 2003. Molecular characterization of rice hsp 101: complementation of yeast hsp104 mutation by disaggregation of protein granules and differential expression in indica and japonica rice types. Plant Mol. Biol., 51: 543-53.

Alessandra C, Stefania DG, Giovanni DB, Antonino C, Marilena C. 2009. Molecular characterization of Italian rice cultivars. Eur. Food Res. Technol., 6: 875-882.

Allen GC, Flores-Vergara MA, Krasynanski S, Kumar S. 2006. A modified protocol for rapid DNA isolation from plant tissues using cetyltrimethylammonium bromide. Nat. Protoc., 1: 2320-2325.

Anuradha A, Kamal K, Pandae KK, Harpal S, Mewari HS, Pankaj P. 2009. Application of RAPD, isozyme and protein markers for characterization of rice (Oryza sativa L.) varieties. Nat. Sc., 7: 795-805.

Cota-Sanchez H, Remarchuk K, Ubayasenaj U. 2006. Ready-touse DNA extracted with a CTAB method adapted for herbarium specimens and mucilaginous plant tissue. Plant Mol. Biol. Rep., 24: 161-167.

Das BK, Jena RC, Samal KC. 2009. Optimization of DNA isolation and PCR protocol for RAPD analysis of banana / plantain (Musa spp.). Int. J. Agri. Sc., 1: 21-25.

Kanawapee N, Sanitchon J, Srihaban P, Theerakulpisut P. 2011. Genetic diversity analysis of rice cultivars (Oryza sativa L.) differing in salinity tolerance based on RAPD and SSR markers. Elect. J Biotech., 6: 25-31.

Karunagoda RP, Bandara CS. 2005. Molecular characterization of some consumer-preferred rice varieties using randomly amplified polymorphic dnas (RAPDs). Sri Lankan J. Agric. Sci., 42: $71-80$.

Liu G, Bernhardt J, Jia MH, Wamishe Y, Jia Y. 2007. Molecular characterization of rice recombinant inbred line population derived from a japonica-indica cross. Euphytica. 159: 73-82.

Mani P, Bastin J, Arun kumar R, Ahmed ABA. 2010. RAPDanalysis of genetic variation of four important rice varieties using two OPR primers. ARPN J. Agric. Biol. Sc., 5: 25-31.
Prabakaran A, Paramasivam K, Rajesh T, Rajarajan D. 2010. Molecular characterization of rice land races using SSR markers. Elec. J. Plant Breed., 1: 512-516.

Qian W, Song G, Yuan HD. 2006. Genetic diversity in accessions of wild rice Oryza granulate from South and Southeast Asia. China Genet. Res. Crop Evol., 53(1): 197204.

Raghunathachari P, Khanna VK, Singh US, Singh NK. 2000. RAPD analysis of genetic variability in Indian scented rice germplasm (Oryza sativa L.). Curr. Sci., 79: 994-998.

Rahman MM, Rahman L, Begum SN, Nur F. 2009. Molecular characterization and genetic diversity study in $\mathrm{F}_{3}$ population of rice. Progress. Agric., 20: 1-8.

Rahman SN, Islam MS, Alam S, Nasiruddin KM. 2007. Genetic polymorphism in rice (Oryza sativa L.) through RAPD analysis. Ind. J. Biotech., 6: 224-229.

Rasheed S, Fatima T, Husnain T, Bashir K, Riazuddin S. 2005. RAPD characterization of somaclonal variation in indica basmati rice. Pak. J. Bot., 37: 249-262.

Saker MM, Youssef SS, Abdallah NA, Bashandy HS, Sharkawy AME. 2005. Genetic analysis of some Egyptian rice genotypes using RAPD, SSR and AFLP. African J. Biotech., 4: 882-890.

Shivapriya M, Shailaja H. 2006. Detection of genotype specific fingerprints and molecular diversity of selected Indian locals and landraces of rice (Oryza sativa L.) using DNA markers. Indian J. Genet. Plant Breed., 66(1): 1-5.

Singh, A. 2003. Genetic analysis of rice (Oryza spp.) accession using Randomly Amplified Polymorphic DNA (RAPD) and microsatellites. Trop. Agric. Res., 15: 77-86.

Tehrim S, Pervaiz ZH, Rabbani MA. 2012. Molecular characterization of traditional and improved rice cultivars based on random amplified polymorphic DNAs (RAPDs) markers. African J. Biotech., 11: 10297-10304.

Thomas G, Mohapatra T, Rao AR, Sharma, R. P. 2006. Distinguishing Indian commercial wheat varieties using RAPD based DNA fingerprints. Indian J. Biotechnol. 5: 200-206.

Yasmin F, Islam MR, Rehana S, Mazumder RR, Anisuzzaman M, Khatun H, Rayhan R, Gregorio GB. 2012. Molecular characterization of inbred and hybrid rice genotypes of Bangladesh. SABRAO J. Breed. Genet., 44: 163-175. 